\title{
ASSOCIATION OF EEG ABNORMALITY AND DEVELOPMENTAL DELAY IN PHENYLKETONURIA (PKU): AN ANALYTIC HISTORICAL CASE CONTROL STUDY
}

\section{P. Karimzadeh}

Pediatric Neurology, Shahid Beheshti University of Medical Sciences(SBMU), Tehran, Iran

Background: Electroencephalogram (EEG) is an easy and non invasive evaluation method for diagnosis and early prognosis in children. Our aim was to assess the association between EEG and the patients' Developmental Quotient (DQ) level in phenylketonuria.

Materials \& methods: In this study, 94 PKU patients (mean age: $8.5 \pm 6.2$ years) who were diagnosed through newborn screening tests or later were followed. PKU was confirmed with a serum phenylalanine concentration above $6 \mathrm{mg} / \mathrm{dl}$ in untreated newborns. The patients were matched in gender, age and phenylalanine level. The ASQ (Age and Stage Questionnaire) was used for evaluation of the developmental stage of the case (abnormal EEG) and control (normal EEG) groups and the Wechsler Intelligence Scale was used to assess cognitive and intellectual abilities. Finally, one way ANOVA and chi square tests were used for analysis and $\mathrm{P}<0.05$ was considered significant.

Results: The case group consisted of 63 patients (67\%) with abnormal EEGs and the control group consisted of 31 patients (33\%) with normal EEGs. In patients with abnormal EEGs, 34 (53\%) had mild, nine (14\%) had moderate and $20(33 \%)$ had severe EEG changes. Distribution of high and low DQ levels in these three groups did not show any significant difference $(\mathrm{p}=0.50)$. Distribution of DQ level in the abnormal and normal EEG patients showed a significant difference $(\mathrm{p}=0.001)$.

Conclusion: There was no significant difference between phenylalanine level in case and

control groups; therefore, EEG findings may affect patients' developmental scores despite a normal phenylalanine level in PKU patients. 